\title{
Anti-FFBRST Transformations for the BLG Theory in Presence of a Boundary
}

\author{
Mir Faizal ${ }^{a *}$, Sudhaker Upadhyay ${ }^{b \dagger}$, Bhabani P Mandal ${ }^{c \ddagger}$ \\ ${ }^{a}$ Department of Physics and Astronomy, University of Waterloo, \\ Waterloo, Ontario N2L 3G1, Canada \\ ${ }^{b}$ Department of Physics, Indian Institute of Technology Kanpur, Kanpur 208016, India \\ ${ }^{c}$ Department of Physics, Banaras Hindu University, Varanasi 221005, India
}

\begin{abstract}
In this paper we will analyse the anti-BRST symmetries of Bagger-Lambert-Gustavsson (BLG) theory in presence of a boundary. We will analyses these symmetries in both linear and non-linear gauges. We will also derive the finite field version of the anti-BRST transformations for the BLG theory in presence of a boundary. These finite field transformations will be used to relate generating functional in linear gauge to the generating functional in the non-linear gauge.
\end{abstract}

\section{Introduction}

According to the AdS/CFT correspondence the superconformal field theory describing multiple M2branes is dual to the eleven dimensional supergravity on $A d S_{4} \times S^{7}$. Now apart from a constant closed 7-form on $S^{7}, A d S_{4} \times S^{7} \sim[S O(2,3) / S O(1,3)] \times[S O(8) / S O(7)] \subset O S p(8 \mid 4) /[S O(1,3) \times S O(7)]$ [1], so the superconformal field theory dual to the eleven dimensional supergravity has $\mathcal{N}=8$ supersymmetry. This is because for this dual superconformal field theory $O S p(8 \mid 4)$ gets realized as $\mathcal{N}=8$ supersymmetry. This superconformal field theory contains eight gauge valued scalar fields which originate from the transversal coordinates of M2-branes. It also contains sixteen physical fermions. Furthermore, it only has sixteen on shell degrees of freedom and so the gauge fields cannot have any contribution to the on shell degrees of freedom. These requirements are met by a theory called the Bagger-Lambert-Gustavsson (BLG) theory [2]-6]. This theory is a three dimensional superconformal field theory with $\mathcal{N}=8$ supersymmetry and $S O(8)$ R-symmetry.

The BLG theory has gauge symmetry and is valued in a Lie 3-algebra rather than a conventional Lie algebra. The only known example of a Lie 3-algebra with fully antisymmetric structure constants is $S O(4)$ and it has not been possible to increase the rank of the gauge group. It is possible to decompose the gauge group $S O(4)$ into $S U(2) \times S U(2)$, by complexifing the matter fields. The gauge sector of the BLG theory now comprises of two Chern-Simons theories with levels $\pm k$ and the matter fields exist in the bi-fundamental representation of the gauge group $S U(2) \times S U(2)$. However, the gauge symmetry is now produced by ordinary Lie algebra rather than a Lie 3-algebra. As the gauge group of the BLG theory is $S U(2) \times S U(2)$, it only represents two M2-branes. It has been possible to extend the gauge group to $U(N) \times U(N)$, and the resultant theory is called Aharony-Bergman-Jafferis-Maldacena (ABJM)

\footnotetext{
*f2mir@uwaterloo.ca

†sudhakerupadhyay@gmail.com

‡bhabani.mandal@gmail.com
} 
theory [7. The ABJM theory only has $\mathcal{N}=6$ supersymmetry, and this supersymmetry gets enhanced to $\mathcal{N}=8$ supersymmetry only for Chern-Simons levels, $k=1,2[9]$. Thus, the ABJM theory only has $\mathcal{N}=6$ supersymmetry instead of $\mathcal{N}=8$ supersymmetry, which M2-branes are expected to have from the $A d S / C F T$ correspondence. However, for two M2-branes ABJM theory coincides with the BLG theory and thus has $\mathcal{N}=8$ supersymmetry.

Just as strings can end on D-branes in string theory, M2-branes can end on other objects in M-theory. Thus, M2-branes can end on M5-branes, M9-branes and gravitational waves. In fact, a form of noncommutative string theory on the M5-brane world-volume has been studied by analyzing the action of a single open M2-branes ending on it [10]-[12. Furthermore, the BLG theory has been used for constructing a novel quantum geometry on the M5-brane world-volume by analysing a system of M2-branes ending on a M5-brane with a constant $C$-field [13. The BLG action with Nambu-Poisson 3-bracket has been identified as the M5-brane action with a large world-volume C-field [14. As the BLG theory has been used for analyzing a system of M2-branes ending on a M5-brane, it is is important to study the BLG theory in presence of a boundary.

In a supersymmetric theory, the presence of a boundary breaks the supersymmetry. This is because the boundary obviously breaks translational symmetry and since supersymmetry closes on translations, it is inevitable that the presence of boundary will also break the supersymmetry. However, half of the the supersymmetry can be preserved by adding a boundary term to the bulk action, such that the supersymmetric variation of this boundary term exactly cancels the boundary piece generated by the supersymmetric transformation of the bulk action [15]-[17. This has been used for analysing the open M2-branes in the ABJM theory [18]-19]. This has also been used for studding the BLG theory in presence of a boundary [20]-21].

It may be noted that the BLG theory in presence of a boundary can be made gauge invariant by adding extra boundary degrees of freedom to it 20. Thus, the boundary BLG also has a gauge symmetry associated with it. So, it cannot be quantized without getting rid of these unphysical degrees of freedom. This can be done by adding ghost and gauge fixing terms to the original classical Lagrangian. The addition of these terms incorporates the gauge fixing condition at a quantum level. It is known that for a gauge theory the sum of the original classical Lagrangian with the gauge fixing and the ghost terms, is invariant under new sets of transformations called the BRST and the anti-BRST transformations [22]25. For the ABJM theory, the BRST and the anti-BRST symmetries have been studied in both linear and non-linear gauges 8 .

The infinitesimal BRST and the infinitesimal anti-BRST transformations have been generalized to finite field dependent BRST (FFBRST) and finite field dependent anti-BRST (anti-FFBRST) transformations [26- 27. This is done by first making the infinitesimal global parameter occurring in the BRST or the anti-BRST transformations depend on fields occurring in the theory. Then this field dependent parameter is integrated to obtain the FFBRST and anti-FFBRST transformations. Even though, these finite transformations are a symmetry of the quantum action, they are not a symmetry of the functional measure. They can thus be used to relate a theory in one gauge to the same theory in a different gauge 28]-32. So, FFBRST transformations can be used to overcome a problem that a theory suffers from in a particular gauge. For example, the axial gauge even for ordinary gauge theories has problamatic poles associates with it. Furthermore, in the Coulomb gauge the time-like propagator is not damped and so the time integral does not converge. There is no reason not to expect similar problems to occur in non-covariant gauges in the BLG theory. However, for ordinary gauge theories these problems can be resolved by using the FFBRST formalism. This is because to overcome these type of difficulties calculation for the required quantity can be done for a gauge in which that problem does not exist, and then using the FFBRST transformation to transform it to the required gauge. In fact, in ordinary Yang-Mills theory, FFBRST transformations have been used for obtaining the propagator in Coulomb gauge from the generating function in the Lorentz gauge [33. Thus, FFBRST can be used for obtaining the propagators in suitable gauges for the ABJM theory, which can be used for calculating scattering amplitudes in a suitable gauge. 
It may be noted that even though the BLG is a theory dual to supergravity theories, and this duality fixes the matter content of the theory, it is a gauge theory from the field theoretic perspective. Thus, at a quantum level it can be analysed as a gauge theory using the Gribov-Zwanziger theory. This way non-perturbative aspects of this theory can be analysed. There is a close relation between FFBRST transformation and Gribov-Zwanziger theory 34. Thus, FFBRST transformations may give us an idea about the non-perturbative effects in a theory. It may be noted that the FFBRST symmetry relies crucially on the gauge sector of the theory, and will not get effected by the matter content of the theory. Hence, even though the BLG theory has a very precise matter content fixed from supergravity theories, the FFBRST formalism can be applied to it. This is very important from the M-theory point of view. This is because we may be able to understand the physics of multiple M5-branes by analysing non-perturbation effects in the BLG theory [35]-38. The FFBRST symmetry for the BLG theory on a manifold without a boundary has already been studied [39. However, so far anti-FFBRST symmetry for BLG theory has not been studied. Furthermore, as the M2-branes can end on M5-branes, it is important to study the BLG theory in presence of a boundary. So, in this paper we will study the anti-FFBRST transformations for the BLG theory in presence of a boundary.

\section{BLG Theory in Presence of a Boundary}

In this section we will review the BLG theory in presence of a boundary [20]-21. As has been demonstrated in the previous section, we have to add a boundary term to the conventional BLG theory to preserve half of the supersymmetry. In fact, we have to add yet another term to it to make it gauge invariant. However, unlike regular gauge theories, where the gauge fields take values in a Lie algebra, the gauge field in the BLG theory take values in a Lie 3-algebra. A Lie 3-algebra is vector space endowed with a trilinear product, $\left[T^{A}, T^{B}, T^{C}\right]=f_{D}^{A B C} T^{D}$, where $T^{A}$ are called the generators of this Lie 3 -algebra. These structure constants are totally antisymmetric in $A, B, C$ and satisfy the Jacobi identity,

$f_{G}^{[A B C} f_{H}^{D] E G}=0$. It is also useful to define the following constants, $C_{E F}^{A B, C D}=2 f_{[E}^{A B[C} \delta_{F]}^{D]}[40]$. These constants are anti-symmetric in the pair of indices $A B$ and $C D$ and the satisfy the following Jacobi identity, $C_{E F}^{A B, C D} C_{K L}^{G H, E F}+C_{E F}^{G H, A B} C_{K L}^{C D, E F}+C_{E F}^{C D, G H} C_{K L}^{A B, G H}=0$. Furthermore, the metric in the Lie 3-algebra can be written as $h_{A B}=\operatorname{Tr}\left(T_{A} T_{B}\right)$.

The BLG theory on manifolds without boundaries has $\mathcal{N}=8$ supersymmetry. However, we will perform our analysis in $\mathcal{N}=1$ superspace formalism where only the supersymmetry generated by $Q_{a}=$ $\partial_{a}-\left(\gamma^{\mu} \partial_{\mu} \theta\right)_{a}$ is manifest. To write the BLG Lagrangian in presence of a boundary, we will first define covariant derivatives for matter fields $X_{A}, X_{A}^{\dagger}$ and the spinor field $\Gamma_{A B}^{a}$ as [40]

$$
\begin{aligned}
\nabla_{a} X_{A}^{I} & =D_{a} X_{A}-i f_{A}^{B C D} \Gamma_{a B C} X_{D}^{I}, \\
\nabla_{a} X_{A}^{I \dagger} & =D_{a} X_{A}^{I \dagger}+i f_{A}^{B C D} X_{D}^{I \dagger} \Gamma^{a B C}, \\
\left(\nabla_{a} \Gamma_{b}\right)_{A B} & =D_{a} \Gamma_{b A B}+C_{A B}^{C D, E F} \Gamma_{C D a} \Gamma_{b E F},
\end{aligned}
$$

where $D_{a}=\partial_{a}+\left(\gamma^{\mu} \partial_{\mu}\right)_{a}^{b} \theta_{b}$. Under gauge transformations these fields transform as $\Gamma_{a} \rightarrow i u \nabla_{a} u^{-1}, X^{I} \rightarrow$ $u X^{I}, X^{I \dagger} \rightarrow X^{I \dagger} u^{-1}$, where $X^{I}=X_{A}^{I} T^{A}, X^{I \dagger}=X_{A}^{I \dagger} T^{A}, \Gamma_{a}=\Gamma_{a A B} T^{A} T^{B}$. We also define, $\Gamma^{a} \times \Gamma_{a}=$ $T^{E} T^{F} C_{E F}^{A B, C D} \Gamma_{A B}^{a} \Gamma_{a C D}$. Now we define,

$$
\begin{aligned}
\Omega_{a A B}= & \omega_{a A B}-\frac{1}{3} C_{A B}^{C D, E F}\left[\Gamma^{b C D}, \Gamma_{a b E F}\right] \\
\omega_{a A B}= & \frac{1}{2} D^{b} D_{a} \Gamma_{b A B}-i C_{A B}^{C D, E F}\left[\Gamma_{C D}^{b}, D_{b} \Gamma_{a E F}\right] \\
& -\frac{1}{3} C_{A B}^{C D, E F} C_{E F}^{G H, I J}\left[\Gamma_{C D}^{b},\left\{\Gamma_{b G H}, \Gamma_{a I J}\right\}\right], \\
\Gamma_{a b A B}= & -\frac{i}{2}\left[D_{(a} \Gamma_{b) A B}-2 i C_{A B}^{C D, E F}\left\{\Gamma_{a C D}, \Gamma_{b E F}\right\}\right] .
\end{aligned}
$$


The Lagrangian for the BLG theory on a manifold without a boundary can be written as 39.

$$
\begin{aligned}
\mathcal{L}= & \nabla^{2}\left[\frac{k}{4 \pi} f^{A B C D} \Gamma_{A B}^{a} \Omega_{a C D}+\frac{1}{4}\left(\nabla^{a} X^{I}\right)^{A}\left(\nabla_{a} X_{I}^{\dagger}\right)_{A}\right. \\
& \left.-\frac{2 \pi}{k} \epsilon_{I J K L} f^{A B C D} X_{A}^{I} X_{B}^{K \dagger} X_{C}^{J} X_{D}^{L \dagger}\right]_{\theta=0} .
\end{aligned}
$$

Now we project the covariant derivatives $\nabla_{ \pm b}=\left(P_{ \pm}\right)_{b}^{a} \nabla_{a}$ using the a projection operator, $\left(P_{ \pm}\right)_{a}^{b}=$ $\left(\delta_{a}^{b} \pm\left(\gamma^{3}\right)_{a}^{b}\right) / 2$. Under the action of this projection operator $Q_{a}$ splits into $Q_{ \pm b}$, where $Q_{ \pm b}=\left(P_{ \pm}\right)_{b}^{a} Q_{a}$ [15]-17. If we put a boundary at fixed $x_{3}$, then $\mu$ splits into $\mu=(\mu, 3)$, and only the supersymmetry generated by one of these supercharges can be preserved. The induced value of the matter and spinor fields on the boundary is denoted by $X_{A}^{\prime}, X_{A}^{\prime}{ }^{\dagger}$ and $\Gamma_{A B}^{a}{ }^{\prime}$, respectively. Furthermore, the boundary covariant derivative of these induced fields, $\nabla_{a}^{\prime}$, is obtained from $\nabla_{a}$, by neglecting $\gamma^{3} \partial_{3}$ contributions to it. We also introduce a boundary superfield $v^{\prime}$ and let $v$ be its extension into the bulk with the following gauge transformation, $v \rightarrow v u^{-1}$. The Lagrangian for the boundary BLG theory which is invariant under supersymmetry generated by $Q_{+}$can be written as follows [20]-21]

$$
\mathcal{L}_{s g}=-\nabla_{+}^{\prime}\left[\mathcal{C S}\left(\Gamma^{v}\right)+\mathcal{M}\left(X^{I}, X^{\dagger I}\right)+\mathcal{K}^{\prime}\left(\Gamma^{\prime}, v^{\prime}\right)\right]_{\theta_{-}=0},
$$

where $\Gamma_{a}^{v}$ denote the gauge transformation of $\Gamma_{a}$ generated by $v$ and

$$
\begin{aligned}
\mathcal{C S}(\Gamma)= & \frac{k}{4 \pi} \nabla_{-}\left[f^{A B C D} \Gamma_{A B}^{a} \Omega_{a C D}\right]_{\theta_{+}=0}, \\
\mathcal{M}\left(X^{I}, X^{\dagger I}\right)= & \frac{1}{4} \nabla_{-}\left[\left(\nabla^{a} X^{I}\right)^{A}\left(\nabla_{a} X_{I}^{\dagger}\right)_{A}\right]_{\theta_{+}=0} \\
& -\frac{2 \pi}{k} \nabla_{-}\left[\epsilon_{I J K L} f^{A B C D} X_{A}^{I} X_{B}^{K \dagger} X_{C}^{J} X_{D}^{L \dagger}\right]_{\theta_{+}=0}, \\
\mathcal{K}^{\prime}\left(\Gamma^{\prime}, v^{\prime}\right)= & -\frac{k}{2 \pi}\left[f_{A B C D}\left(v^{\prime-1} \nabla_{+}^{\prime} v^{\prime}\right)^{A B}\left(v^{\prime-1} \mathcal{D}_{-}^{\prime} v^{\prime}\right)^{C D}\right]_{\theta_{+}=0} .
\end{aligned}
$$

It may be noted that $\mathcal{S}\left(\Gamma^{\prime}, v^{\prime}\right)=\mathcal{C S}\left(\Gamma^{v}\right)-\mathcal{C S}(\Gamma)$ is the boundary potential. So, $\mathcal{C} \mathcal{S}\left(\Gamma^{v}\right)=\mathcal{C} \mathcal{S}(\Gamma)+$ $\left.\mathcal{S}\left(\Gamma^{\prime}, v^{\prime}\right)\right)$ is the total potential of the theory. In case there is no coupling to the bulk fields this reduces to a potential term for the supersymmetric Wess-Zumino-Witten models,

$$
\begin{aligned}
\nabla_{+}^{\prime} \mathcal{S}\left(\Gamma^{\prime}, v^{\prime}\right)= & -\frac{k}{2 \pi} \nabla_{+}^{\prime} C_{A B}^{C D, E F}\left[\left[\left(v^{-1^{\prime}} \mathcal{D}_{-}^{\prime} v^{\prime}\right)^{A B},\left(v^{-1^{\prime}} \mathcal{D}_{3}^{\prime} v^{\prime}\right)_{C D}\right]\right. \\
& \left.\times\left(v^{-1^{\prime}} \nabla_{+}^{\prime} v^{\prime}\right)_{E F}\right]_{\theta_{-}=0}
\end{aligned}
$$

\section{Finite Field Dependent Transformation}

In this section we will construct finite field dependent anti-BRST transformations for the BLG theory in presence of a boundary. To do that we first note that we can write the gauge fixing term for the BLG theory as

$$
\mathcal{L}_{g f}=\nabla_{+} \nabla_{-}\left[f^{A B C D} b_{A B} D^{a} \Gamma_{a C D}+\frac{\alpha}{2} f^{A B C D} b_{A B} b_{C D}\right]_{\theta=0} .
$$

The ghost term corresponding to this gauge fixing term can be written as

$$
\mathcal{L}_{g h}=\nabla_{+} \nabla_{-}\left[f^{A B C D} \bar{c}_{A B} D^{a} \nabla_{a} c_{C D}\right]_{\theta=0} .
$$

The total Lagrangian, $\mathcal{L}_{B L G}=\mathcal{L}_{s g}+\mathcal{L}_{g f}+\mathcal{L}_{g h}$, is now invariant under the following anti-BRST transformations,

$$
\bar{s} \Gamma_{a}=\nabla_{a} \bar{c}, \quad \bar{s} \bar{c}=-\bar{c} \times \bar{c},
$$




$$
\begin{aligned}
\bar{s} X^{I \dagger}=-i X^{I \dagger} \bar{c}, & \bar{s} b=-b \times \bar{c}, \\
\bar{s} X^{I}=i \bar{c} X^{I}, & \bar{s} c=-b-c \times c, \\
\bar{s} v=-i v \bar{c} . &
\end{aligned}
$$

In fact, it is also possible to write a non-linear parts of the above anti-BRST transformations as

$$
\begin{aligned}
\bar{s} c & =-b-\frac{1}{2} \bar{c} \times c, \\
\bar{s} b & =-\frac{1}{2} \bar{c} \times b+\frac{1}{8} \bar{c} \times \bar{c} \times c, \\
\bar{s} \bar{c} & =-\frac{1}{2} \bar{c} \times \bar{c} .
\end{aligned}
$$

Now the infinitesimal global parameter had to be included in the definition of $\bar{s}$. However, we can explicitly write this global parameter with odd Grassmann parity occurring in these transformations as $\epsilon$. The properties of the anti-BRST transformation do not depend on whether this parameter is field dependent or not. Similarly, they do not depend on whether this parameter is finite or infinitesimal. Thus, we first make this infinitesimal global parameter with odd Grassmann parity an infinitesimal field dependent parameter with odd Grassmann parity $\epsilon[\Phi(x, \kappa)]$, where $\Phi^{i}(x, \kappa)=\left(X^{I}(x, \kappa), X^{I^{\dagger}}(x, \kappa), \Gamma_{a}(x, \kappa), c(x, \kappa), \bar{c}(x, \kappa), b(x, \kappa), v(x, \kappa)\right)$. Here $\Phi^{i}(x, 0)$ are the initial fields and $\Phi^{i}(x, 1)$ are the transformed field. Furthermore, $\kappa: 0 \leq \kappa \leq 1$ is an arbitrary parameter and physical quantities do not depend on it [41, 26].

Now we can make this infinitesimal field dependent parameter a finite field dependent parameter and thus define a functional with odd Grassmann parity as $\Theta[\Phi]$. This finite field dependent parameter can obtained from a infinitesimal field dependent parameter as follows,

$$
\Theta[\Phi(x, \kappa)]=\epsilon[\Phi(x, \kappa)] \frac{\exp F[\Phi(x, \kappa)]-1}{F[\Phi(x, \kappa)]},
$$

where

$$
\begin{aligned}
F= & \frac{\delta \epsilon[\Phi(x, \kappa)]}{\delta X^{I}(x, \kappa)} \bar{s} X^{I}(x, \kappa)+\frac{\delta \epsilon[\Phi(x, \kappa)]}{\delta X^{I \dagger}(x, \kappa)} \bar{s} X^{I^{\dagger}}(x, \kappa)+\frac{\delta \epsilon[\Phi(x, \kappa)]}{\delta \Gamma_{a}(x, \kappa)} \bar{s} \Gamma_{a}(x, \kappa) \\
& +\frac{\delta \epsilon[\Phi(x, \kappa)]}{\delta c(x, \kappa)} \bar{s} c(x, \kappa)+\frac{\delta \epsilon[\Phi(x, \kappa)]}{\delta \bar{c}(x, \kappa)} \bar{s} \bar{c}(x, \kappa)+\frac{\delta \epsilon[\Phi(x, \kappa)]}{\delta b(x, \kappa)} \bar{s} b(x, \kappa) \\
& +\frac{\delta \epsilon[\Phi(x, \kappa)]}{\delta v(x, \kappa)} \bar{s} v(x, \kappa) .
\end{aligned}
$$

For a field dependent infinitesimal parameter $\epsilon[\Phi(x, \kappa)]$, we have

$$
\frac{d}{d \kappa} \Phi^{i}(x, \kappa)=\bar{s} \Phi^{i}(x, \kappa) \epsilon[\Phi(x, \kappa)] .
$$

We integrate these equations from $\kappa=0$ to $\kappa=1$ and find the relation between $\Phi^{i}(x, 1)$ and $\Phi^{i}(x, 0)$,

$$
\Phi^{i}(x, 1)=\Phi^{i}(x, 0)+\bar{s} \Phi^{i}(x, 0) \Theta[\Phi(x)],
$$

Now the anti-FFBRST transformations for the BLG theory are given by $f \Phi^{i}=\bar{s} \Phi^{i} \Theta$. The anti-FFBRST transformations are a symmetry of the action $S_{B L G}$. However, they are not a symmetry of the functional measure because the Jacobian for path integral measure in the expression of generating functional is not invariant under them. The change in the Jacobian under the FFBRST transformation is given by $\mathcal{D} \Phi^{i}=J[\Phi(\kappa)] \mathcal{D} \Phi^{i}(\kappa)$. Now $J[\Phi(\kappa)]$ can be replaced within the functional integral by $e^{i S_{1}[\Phi(\kappa)]}$, where $S_{1}[\Phi(\kappa)]$ is some local functional of $\Phi^{i}$. The condition for existence of $S_{1}$ for the BLG theory in presence of a boundary is given by

$$
\int d^{3} x \nabla_{+} \nabla_{-}\left[\frac{1}{J(\kappa)} \frac{d J(\kappa)}{d \kappa}-i \frac{d S_{1}}{d \kappa}\right]_{\theta=0}=0
$$


The infinitesimal change in Jacobian is given by

$$
\delta \mathcal{J}(\kappa)=\nabla_{+} \nabla_{-}\left[\frac{1}{J(\kappa)} \frac{d J(\kappa)}{d \kappa}\right]_{\theta=0} .
$$

So, we can write

$$
\begin{aligned}
\delta \mathcal{J}(\kappa)= & -\int d^{3} x \nabla_{+} \nabla_{-}\left[-\bar{s} X^{I}(x, k) \frac{\delta \epsilon[\Phi(x, k)]}{\delta X^{I}(x, k)}\right. \\
& -\bar{s} X^{I \dagger}(x, k) \frac{\delta \epsilon[\Phi(x, k)]}{\delta X^{I \dagger}(x, k)}+\bar{s} \Gamma_{a}(x, k) \frac{\delta \epsilon[\Phi(x, k)]}{\delta \Gamma_{a}(x, k)} \\
& -\bar{s} c(x, k) \frac{\delta \epsilon[\Phi(x, k)]}{\delta c(x, k)}-\bar{s} \bar{c}(x, k) \frac{\delta \epsilon[\Phi(x, k)]}{\delta \bar{c}(x, k)} \\
& \left.+\bar{s} b(x, k) \frac{\delta \epsilon[\Phi(x, k)]}{\delta b(x, k)}+\bar{s} v(x, k) \frac{\delta \epsilon[\Phi(x, k)]}{\delta v(x, k)}\right]_{\theta=0} .
\end{aligned}
$$

\section{Anti-FFBRST Transformations with specific parameter}

We will now use anti-FFBRST to relate the BLG theory, in the linear gauge to it in the non-linear gauge. Here the linear anti-BRST transformations are denoted by $\left(\bar{s} G_{1}\right)^{A B}$ and the the non-linear anti-BRST transformations are denoted by $\left(\bar{s} G_{2}\right)^{A B}$. We again define the infinitesimal field dependent parameter as follows

$$
\epsilon[\Phi]=-i \gamma \int d^{3} x \nabla_{+} \nabla_{-}\left[f^{A B C D} c_{A B}\left(G_{C D 1}-G_{C D 2}\right)\right]_{\theta=0},
$$

where $\gamma$ is an arbitrary constant parameter. However, the expression for the change in Jacobian is now given by

$$
\begin{aligned}
\delta \mathcal{J}(\kappa)= & i \gamma \int d^{3} x \nabla_{+} \nabla_{-} f^{A B C D}\left[\left(\bar{s} G_{C D 1}-\bar{s} G_{C D 2}\right) c_{A B}\right. \\
& \left.+\left(b_{A B}+C_{A B}^{G H, E F} \bar{c}_{G H} c_{E F}\right)\left(G_{C D 1}-G_{C D 2}\right)\right]_{\theta=0}
\end{aligned}
$$

Furthermore, we construct the local functional as

$$
\begin{aligned}
S_{1}= & \int d^{3} x \nabla_{+} \nabla_{-} f^{A B C D}\left[\xi_{1}(\kappa) b_{A B} G_{C D 1}+\xi_{2}(\kappa) b_{A B} G_{C D 2}\right. \\
& +\xi_{3}(\kappa) \bar{s} G_{C D 1} c_{A B}+\xi_{4}(\kappa) \bar{s} G_{C D 2} c_{A B} \\
& +\xi_{5}(\kappa) C_{A B}^{G H, E F} \bar{c}_{G H} c_{E F} G_{C D 1} \\
& \left.+\xi_{6}(\kappa) C_{A B}^{G H, E F} \bar{c}_{G H} c_{E F} G_{C D 2}\right]_{\theta=0},
\end{aligned}
$$

where $\xi_{i}(i=1,2,3,4)$ are the $\kappa$ dependent arbitrary parameters which satisfy the following initial boundary condition $\xi_{i}(\kappa=0)=0$. As all the fields again depend on $\kappa$, so we calculate

$$
\begin{aligned}
\frac{d S_{1}}{d \kappa}= & \int d^{3} x \nabla_{+} \nabla_{-} f^{A B C D}\left[\xi_{1}^{\prime} b_{A B} G_{C D 1}-\xi_{1} C_{A B}^{G H, E F} b_{G H} \bar{c}_{E F} G_{C D 1} \epsilon\right. \\
& +\xi_{1} b_{A B} \bar{s} G_{C D 1} \epsilon+\xi_{2}^{\prime} b_{A B} G_{C D 2}-\xi_{2} C_{A B}^{G H, E F} b_{G H} \bar{c}_{E F} G_{C D 2} \epsilon \\
& +\xi_{3}^{\prime} \bar{s} G_{C D 1} c_{A B}-\xi_{3} \bar{s} G_{C D 1}\left(b_{A B}+C_{A B}^{G H, E F} \bar{c}_{G H} c_{E F}\right) \epsilon \\
& +\xi_{4}^{\prime} \bar{s} G_{C D 2} c_{A B}-\xi_{4} \bar{s} G_{C D 2}\left(b_{A B}+C_{A B}^{G H, E F} \bar{c}_{G H} c_{E F}\right) \epsilon \\
& +\xi_{5}^{\prime} C_{A B}^{G H, E F} \bar{c}_{G H} c_{E F} G_{C D 1}+\xi_{6}^{\prime} C_{A B}^{G H, E F} \bar{c}_{G H} c_{E F} G_{C D 2}
\end{aligned}
$$




$$
\begin{aligned}
& -\xi_{5} C_{A B}^{G H, E F} \bar{c}_{G H} b_{E F} G_{C D 1} \epsilon+\xi_{5} C_{A B}^{G H, E F} \bar{c}_{G H} c_{E F} \bar{s} G_{C D 1} \epsilon \\
& -\xi_{6} C_{A B}^{G H, E F} \bar{c}_{G H} b_{E F} G_{C D 2} \epsilon+\xi_{6} C_{A B}^{G H, E F} \bar{c}_{G H} c_{E F} \bar{s} G_{C D 2} \epsilon \\
& \left.+\xi_{2} b_{A B} \bar{s} G_{C D 2} \epsilon\right]_{\theta=0}, \\
= & \int d^{3} x \nabla_{+} \nabla_{-} f^{A B C D}\left[\xi_{1}^{\prime} b_{A B} G_{C D 1}+\xi_{3}^{\prime} \bar{s} G_{C D 1} c_{A B}\right. \\
& +\xi_{5}^{\prime} C_{A B}^{G H, E F} \bar{c}_{G H} c_{E F} G_{C D 1}+\xi_{6}^{\prime} C_{A B}^{G H, E F} \bar{c}_{G H} c_{E F} G_{C D 2} \\
& +\left(\xi_{1}-\xi_{3}\right) \bar{s} G_{C D 1} b_{A B} \epsilon-\left(\xi_{3}-\xi_{5}\right) C_{A B}^{G H, E F} \bar{c}_{G H} c_{E F} \bar{s} G_{C D 1} \\
& +\left(\xi_{2}-\xi_{4}\right) \bar{s} G_{C D 2} b_{A B} \epsilon-\left(\xi_{4}-\xi_{6}\right) C_{A B}^{G H, E F} \bar{c}_{G H} c_{E F} \bar{s} G_{C D 2} \epsilon \\
& -\left(\xi_{1}-\xi_{5}\right) C_{A B}^{G H, E F} b_{G H} \bar{c}_{E F} G_{C D 1} \epsilon+\xi_{4}^{\prime} \bar{s} G_{C D 2} c_{A B} \\
& \left.-\left(\xi_{2}-\xi_{6}\right) C_{A B}^{G H, E F} b_{G H} \bar{c}_{E F} G_{C D 2} \epsilon+\xi_{2}^{\prime} b_{A B} G_{C S 2}\right]_{\theta=0} .
\end{aligned}
$$

Now, the Jacobian can be written as $e^{i S_{1}}$, if the following equation is satisfied

$$
\begin{aligned}
& \int d^{3} x \nabla_{+} \nabla_{-}\left[f ^ { A B C D } \left[\left(\xi_{1}^{\prime}-\gamma\right) b_{A B} G_{C D 1}+\left(\xi_{2}^{\prime}+\gamma\right) b_{A B} G_{C S 2}\right.\right. \\
& +\left(\xi_{5}^{\prime}-\gamma\right) C_{A B}^{G H, E F} \bar{c}_{G H} c_{E F} G_{C D 1}+\left(\xi_{6}^{\prime}+\gamma\right) C_{A B}^{G H, E F} \bar{c}_{G H} c_{E F} G_{C D 2} \\
& +\left(\xi_{1}-\xi_{3}\right) \bar{s} G_{C D 1} b_{A B} \epsilon-\left(\xi_{3}-\xi_{5}\right) C_{A B}^{G H, E F} \bar{c}_{G H} c_{E F} \bar{s} G_{C D 1} \\
& +\left(\xi_{2}-\xi_{4}\right) \bar{s} G_{C D 2} b_{A B} \epsilon-\left(\xi_{4}-\xi_{6}\right) C_{A B}^{G H, E F} \bar{c}_{G H} c_{E F} \bar{s} G_{C D 2} \epsilon \\
& -\left(\xi_{1}-\xi_{5}\right) C_{A B}^{G H, E F} b_{G H} \bar{c}_{E F} G_{C D 1} \epsilon+\left(\xi_{4}^{\prime}+\gamma\right) \bar{s} G_{C D 2} c_{A B} \\
& -\left(\xi_{2}-\xi_{6}\right) C_{A B}^{G H, E F} b_{G H} \bar{c}_{E F} G_{C D 2} \epsilon \\
& \left.+\left(\xi_{3}^{\prime}-\gamma\right) \bar{s} G_{C D 1} c_{A B}\right]_{\theta=0}=0 .
\end{aligned}
$$

So, equating the coefficients of the above expression, we get $\xi_{1}^{\prime}-\gamma=0, \xi_{2}^{\prime}+\gamma=0, \xi_{3}^{\prime}-\gamma=0, \xi_{4}^{\prime}+\gamma=$ $0, \xi_{5}-\gamma=0, \xi_{6}+\gamma=0$, and $\xi_{1}-\xi_{3}=\xi_{2}-\xi_{4}=\xi_{3}-\xi_{5}=\xi_{4}-\xi_{6}=\xi_{1}-\xi_{5}=\xi_{2}-\xi_{6}=0$. For $\gamma=-1$, the solutions to above equations satisfying initial condition for $\xi$ are given by $\xi_{1}=-\kappa, \xi_{2}=\kappa, \xi_{3}=$ $-\kappa, \xi_{4}=\kappa, \xi_{5}=-\kappa, \xi_{6}=\kappa$. Now, by adding $S_{1}(\kappa=1)$ to the original action in the gauge $G_{C D 1}$, we obtain the action in gauge $G_{C D 2}, S_{f}=S_{B L G}+S_{1}$. So, under the anti-FFBRST transformations the generating functional in the gauge $G_{C D 2}$ transforms to the generating functional in the gauge $G_{C D 1}$. In fact, the anti-FFBRST transformations can also be used to obtain the generating functional in the gauge $G_{C D 2}$, if we start from the generating functional in the gauge $G_{C D 1}$.

\section{Conclusion}

In this paper we analysed the BLG theory on a manifold with a boundary. This theory was made gauge invariant by adding a new field to it, such that the boundary term generated by the gauge transformations of the BLG theory exactly canceled the boundary term generated by the gauge transformations of this new term. The measure of integration of the superspace was also modified so that the theory preserves half the supersymmetry even in presence of a boundary. Furthermore, the anti-BRST transformations of this theory were studied in both linear as well as non-linear gauges. After analysing the both the linear and non-linear anti-BRST transformations, the anti-FFBRST transformations were constructed. It was demonstrated that these two gauges can be related to each other via anti-FFBRST transformations.

The main motivation for studding the FFBRST or the anti-FFBRST transformation of the BLG theory is that they are related to Gribov-Zwanziger theory and can thus give us an idea about the non-perturbative effects in the BLG theory. As the physics of multiple M5-branes can be studied using non-perturbation effects in the BLG theory [35]-38, FFBRST transformations for the BLG theory are very important. Furthermore, as M2-branes can end on M5-branes, it is important to study the FFBRST 
transformations for the BLG theory on a manifold with boundaries. However, it may be noted that the BLG theory only describes two M2-branes. It has been generalized to the ABJM theory, which describes more than two M2-branes [7. The BRST and the anti-BRST symmetries for the ABJM theory have been studied in various gauges [8]. Thus, it would be interesting to study the FFBRST and the antiFFBRST transformations for the ABJM theory. The ABJM theory in presence of a boundary has also been constructed [18]-[19]. In fact, the BRST and the anti-BRST transformations for the ABJM theory in presence of a boundary have also been discussed [18. So, it would also be interesting to find a finite field version of these transformations. However, as the ABJM theory has two infinitesimal gauge parameters, the conventional FFBRST transformation would have to be modified for the ABJM theory. It may be noted that the ABJM theory coincides with the BLG theory for two M2-branes. So, for two M2-branes, the FFBRST transformations for the ABJM theory should reduce to the FFBRST transformations for the BLG theory. The FFBRST transformation for gauge symmetry generated by a Lie 3-algebra can used for analysing the FFBRST transformation for the gauge theory with two parameters as is possible to decompose $S O(4)$ into $S U(2) \times S U(2)$. This can then be used to motivate the analysis of FFBRST transformations for the ABJM theory.

It may be noted that multiple D2-brane action can be derived from a multiple M2-brane action by means of a novel Higgs mechanism [42]-45]. In this mechanism a vacuum expectation value is given to a scalar field. This breaks the gauge group of the ABJM theory down to its diagonal subgroup. Thus, the Yang-Mills theory coupled to matter fields is obtained by Higgsing the ABJM theory. It would be interesting to start with a gauge fixed ABJM theory and derive its FFBRST transformations, then using the novel Higgs mechanism to go to the multiple D2-branes action. It would be expected that the FFBRST transformations in this case will reduce to the FFBRST transformations for the D2-branes. By analysing these transformations, we can get a better understanding of what happens to this theory at a quantum level. We can also perform a similar analysis using anti-FFBRST transformations.

It may be noted that for Yang-Mills theory in Cho-Faddeev-Niemi variables with an appropriate choices of finite and field dependent parameter the gauge fixing and ghost terms corresponding to Landau gauge and maximal Abelian gauge appear naturally by using the FFBRST transformations [46]. It will be interesting to perform a similar analysis for M2-branes and show that the gauge fixing and ghost terms corresponding to different gauges can also occur naturally for M2-branes by using the FFBRST transformations. It may be noted that various deformations of the general relativity have been studied [47-[50, and it would be possible to study such deformations of supergravity in eleven dimensions. So, it will be interesting to analyse the action for M2-branes dual to such deformations of supergravity in eleven dimensions, and repeat the analysis of this paper for such deformations.

\section{References}

[1] E. Ivanov, arXiv:0912.5505

[2] A. Gustavsson, JHEP. 0804, 083 (2008)

[3] J. Bagger and N. Lambert, JHEP. 0802, 105 (2008)

[4] J. Bagger and N. Lambert, Phys. Rev. D77, 065008 (2008)

[5] M. A. Bandres, A. E. Lipstein and J. H. Schwarz, JHEP. 0809, 027 (2008)

[6] E. Antonyan and A. A. Tseytlin, Phys. Rev. D79, 046002 (2009)

[7] O. Aharony, O. Bergman, D. L. Jafferis and J. Maldacena, JHEP. 0810, 091 (2008)

[8] M. Faizal, Phys. Rev. D84, 106011 (2011)

[9] O-Kab Kwon, P. Oh and J. Sohn, JHEP. 0908, 093 (2009) 
[10] E. Bergshoeff, D. S. Berman, J. P. van der Schaar and P. Sundell, Nucl. Phys. B 590, 173 (2000)

[11] S. Kawamoto and N. Sasakura, JHEP 0007, 014 (2000)

[12] D. S. Berman and B. Pioline, Phys. Rev. D 70, 045007 (2004)

[13] C. S. Chu and D. J. Smith, JHEP. 0904, 097 (2009)

[14] P. M. Ho, Chin. J. Phys. 48, 1 (2010)

[15] D. V. Belyaev and P. V. Nieuwenhuizen, JHEP. 0804, 008 (2008)

[16] M. Faizal, Int. J. Theor. Phys. 52, 392 (2013)

[17] M. Faizal and D. J. Smith, Phys. Rev. D87, 025019 (2013)

[18] M. Faizal and D. J. Smith, Phys. Rev. D 85, 105007 (2012)

[19] D. S. Berman and D. C. Thompson, Nucl. Phys. B820, 503 (2009)

[20] M. Faizal, JHEP. 1204, 017 (2012)

[21] M. Faizal, Mod. Phys. Lett. A 29, 1450154 (2014)

[22] C. Becchi, A. Rouet and R. Stora, Annals. Phys. 98, 287 (1976)

[23] I. V. Tyutin, Lebedev. preprint. fian. 39 (1975)

[24] D. Dudal, H. Verschelde, V. E. R. Lemes, M. S. Sarandy, S. P. Sorella and M. Picariello, Ann. Phys. $308,62(2003)$

[25] D. Dudal, V.E.R. Lemes, M. Picariello, M.S. Sarandy, S.P. Sorella and H. Verschelde, JHEP. 0212, $008(2002)$

[26] S. D. Joglekar and B. P. Mandal, Phys. Rev. D 51, 1919 (1995)

[27] S. Upadhyay, S. K. Rai and B. P. Mandal, J. Math. Phys. 52, 022301 (2011)

[28] S. D. Joglekar and A. Misra, Int. J. Mod. Phys. A 15, 1453 (2000)

[29] S. D. Joglekar and B. P. Mandal, Int. J. Mod. Phys. A 17, 1279 (2002)

[30] R. Banerjee, B. P. Mandal, Phys.Lett. B 48827 (2000)

[31] S. K. Rai, B. P. Mandal, Eur. Phys. J. C 63, 323 (2009)

[32] S. Upadhyay and B. P. Mandal, Mod. Phys. Lett. A 25, 3347 (2010)

[33] S. D. Joglekar and B. P.Mandal, Int. J. Mod. Phys. A17, 1279 (2002)

[34] S. Upadhyay and B. P. Mandal, Euro. Phys. Lett. 93, 31001 (2011)

[35] S. P. Chowdhury and S. Chakrabortty and K. Ray, Phys. Lett. B703, 172 (2011)

[36] M. A. Ganjali, JHEP. 0903, 064 (2009)

[37] P. M. Ho, Y. Imamura, Y. Matsuo and S. Shiba, JHEP. 0808, 014 (2008)

[38] A. Gustavsson, JHEP. 0911, 071 (2009)

[39] M. Faizal, B. P. Mandal and S. Upadhyay, Phys. Lett. B 721, 159 (2013)

[40] A. Gustavsson, Nucl. Phys. B 807, 315 (2009) 
[41] R. Banerjee and S. Upadhyay, Phys. Lett. B 734, 369 (2014)

[42] S. Mukhi and C. Papageorgakis, JHEP. 0805, 085 (2008)

[43] T. Li, Y. Liu and D. Xie, Int. J. Mod. Phys. A24, 3039 (2009)

[44] Y. Pang and T. Wang, Phys. Rev. D78, 125007 (2008)

[45] P. M. Ho, Y. Imamura and Y. Matsuo, JHEP. 0807, 003 (2008)

[46] S. Upadhyay, Phys. Lett. B 727, 293 (2013)

[47] M. Faizal, J. Phys. A 44, 402001 (2011)

[48] M. Faizal, Mod. Phys. Lett. A 27, 1250075 (2012)

[49] P. Horava, Phys. Rev. D 79, 084008 (2009)

[50] M. Kober, Phys. Rev. D 82, 085017 (2010) 